\title{
Suppressing with Images
}

\author{
Aneta Niczyporuk \\ Institute of Sociology and Cognitive Science \\ University of Bialystok, Poland \\ aneta.niczyporuk@uwb.edu.pl
}

Received 1 September 2018; accepted 23 October 2018; published 18 September 2019

\begin{abstract}
Research reveals a unique relationship between image representations and emotions. In this context, intrusive mental images could be especially unpleasant and distressing. On the other hand, because of their impact on emotions, images can serve as a support to selfregulation, including regulation of one's own thoughts. In the article, a thought suppression strategy is proposed that uses images to fight harmful intrusions. This proposition is based on several research areas: studies on image representations properties, the "think/nothink" and the "white bear" thought suppression paradigms, and Lavie's attention theory. The strategy assumes the redirection of attention from unwanted content by using image representations in conditions of maximally freed-up cognitive control resources.
\end{abstract}

Keywords: thought suppression; intrusions; cognitive control; load; image representations.

\section{Introduction}

To some extent, we can choose how we think - with words or with images - and our choice has consequences. For example, thinking with words is easier to communicate: a verbal thought is somewhat ready to be conveyed to others, while images need to be translated into words, or a perceptual experience needs to be evoked in an interlocutor, e.g. by drawing a picture or singing a melody. Verbal and image thinking also differ in their capacity to evoke emotions. Recent research shows that image representations, in comparison with verbal ones, have a stronger impact on emotional processes. This might be why intrusive images are one of the symptoms of many mental disorders. Currently, there is increased interest in this problem (Brewin, Gregory, Lipton, \& Burgess, 2010). In posttraumatic stress disorder, patients suffer from nightmares and recurrent traumatic images. 
In phobias, there is a problem with intrusive images of a person's fears; for example, people with social phobia might imagine that they are heavily blushing, and those with arachnophobia are haunted by images of spiders. Patients with obsessive-compulsive disorder have to deal with intrusions that illustrate the threat of contamination. Those suffering from depression report the appearance of unpleasant images from the past, and patients with bipolar disorder may experience images of the future, including potential suicide attempts. There is a multitude of examples of intrusive images in mental disorders (for reviews see, for example, Çili \& Stopa, 2015; Holmes \& Mathews, 2010; Pearson, Naselaris, Holmes, \& Kosslyn, 2015).

Healthy people also struggle with intrusive thoughts (Rachman \& de Silva, 1978). Intrusions can perform important functions, for example regulating activity related to an important goal by reminding that goal fulfilment may be at risk. If we look at the examples of intrusive thoughts mentioned above, we can see that they concern important problems that are characteristic of a given disorder. Completely getting rid of intrusive thoughts seems like a very dangerous idea, but certain regulation of intrusions might be helpful and needed. In the case of mental disorders, intrusions are a source of unnecessary suffering and contribute to the disorganization of a patient's life. In the case of people who do not suffer from disorders, we can also find situations in which intrusions do not benefit the person because, for example, they exaggerate a threat.

The aim of the article is to search for a way to effectively and healthily deal with unwanted thoughts, starting with intrusive images. The development of such a method could prove particularly useful for people who are unable on their own to free themselves from the harmful effects of intrusion. Due to the unique relationship between image representations and emotional processes, such a strategy for fighting intrusions could be based on images.

In the article, I try to outline a strategy of suppressing thoughts (mostly but not exclusively in image form) using thinking with images. I broadly understand thinking with images as the processing of sensory information related to the current or imagined stimulus. Before discussing the proposed strategy, I first review research which shows that image representations have a stronger impact on emotional processes. This is why I think that images have the potential to be especially effective in fighting intrusions. Next, I review the research and theory of thought suppression, focusing on two thought suppression paradigms (the "think/no-think" paradigm and the "white bear" paradigm) and Lavie and colleagues' work on attention (which is also related to thought suppression, albeit indirectly). Finally, I outline a proposed strategy of suppressing intrusions and confront it with existing empirical data.

\section{Image Representations and Emotions: Empirical Data}

We can easily list situations in which an image evokes intense emotions. We feel longing when we remember the face of a friend we have not seen in a long time, or we feel sadness and compassion at the sight of needy children in social campaigns. The law has also noticed the role of images in stimulating emotions. For a year now, packets of cigarettes sold 
in the European Union have had to include pictures showing the harmful effects of smoking next to a written warning. On the other hand, a Polish court has recently found antiabortion activists guilty not because of the use of slogans such as "Abortion kills unborn children," but for publicly exhibiting photos of bloody fetuses. Such observations and convictions that images have a particularly strong impact on emotions provided the impetus to conduct experiments that explore the relationship between thinking with images and emotional processes.

One of the first scientific experiments relating to the influence of mental images on emotions was the study of Vrana, Cuthbert, and Lang (1986). The authors asked participants to process previously memorized neutral or distressing sentences verbally (repeating them in their minds) or with images (imagining themselves in the described situation). Image processing of distressing sentences contributed to a greater increase in heart rate than verbal processing. Faster heart rate can be treated as an indicator of emotional arousal, although the study lacked a more direct measurement of this factor, such as self-reported estimation of anxiety level.

Heart rate was also measured in the study of Miller and colleagues (1987), for which in the course of analysis participants were divided into those with higher and lower visualization abilities, as measured with a questionnaire. During the experiment, the participants heard descriptions of different situations: emotionally neutral, containing rapid action, distressing (anxious), and evoking anger. People who visualized better had a faster heart rate when imagining a situation characterized by anger than when imagining other situations; such differences were not detected in people with lower visualization abilities. This may indicate that the images involve more visible emotional reactions, thus differentiating the reception of the described situations.

The works of Holmes and colleagues is of particular importance for understanding the relationship between image representations and emotions. In one of their articles (Holmes $\&$ Mathews, 2005), the increase of anxiety was greater when people imagined threatening situations than when they processed the meaning of the descriptions of the same situations without the use of imagination. In correlational studies on a group of cancer patients, it was also confirmed that intrusive mental images are associated with greater discomfort than verbal (Whitaker, Watson, \& Brewin, 2009).

Imagination also stimulates pleasant affective states more strongly (Holmes, Lang, \& Shah, 2009). An increase in positive affect was observed among participants who imagined situations with a happy ending, while the level of positive affect decreased among participants who processed the described situations in a verbal manner (Holmes, Mathews, Dalgleish, \& Mackintosh, 2006). The expressiveness of mental images depicting positive future events correlates with optimism (Blackwell et al., 2013).

The aforementioned experiments could arouse methodological concerns because the material that was used was presented to all participants verbally; only then were they asked to imagine the presented content or focus on its meaning without the use of their imagination. 
Thus, the results can be explained not by the unique impact of mental images on emotions, but rather by referring (in a group with visualization) to two types of representation: words and images. In response to this problem, a study was conducted in which participants were presented with images with assigned words (Holmes, Mathews, Mackintosh, \& Dalgleish, 2008, experiment 1). Participants were asked to combine a picture with a word. The stimuli were chosen so that combining them would bring to mind a physical threat (for example, the combination of a picture of a person standing on stairs, with the word "fall" associated with the concept of falling from stairs). Next, the participants evaluated the intensity of emotions evoked by picture-word pairs and the way they combined these pairs (by thinking in mental images, by thinking in words, or in some other way). There was a correlation between the degree of involvement of imaginative thinking and the intensity of emerging emotions. There was no correlation between the intensity of emotions and the degree of verbal thinking. In addition, the use of imagination correlated with the appearance of participants' own memories depicting the content of the combinations. In the next experiment (Holmes et al., 2008, experiment 2), participants were instructed on how they should combine stimuli (either by imagination or with sentences). Stimuli with a pleasant emotional overtone were also added. In comparison to the verbal condition, in the imagination condition negative and positive stimuli resulted in a greater increase or decrease in anxiety, respectively.

In conclusion, there is research — although not yet very plentiful - that indicates a special relationship between image representations and emotional states. How can we explain this relationship?

\section{Theoretical Explanation of the Relationship between Images and Emotions}

Holmes and Mathews (2010) offer three non-exclusive mechanisms that can explain how mental images evoke vivid emotional reactions. One of them concerns the relation between perception and imagination. Neuroimaging studies indicate that the brain areas responsible for perception and imagination overlap to a large extent. This might be responsible for the fact that we see mental images as if they were real, which may consequently cause similar emotional reactions to both images. Just as the view of a predator naturally evokes fear, its mental image — which stimulates similar areas of the brain as in perception - can also activate connections associated with an analogous emotional response (Burnett Heyes, Lau, \& Holmes, 2013). Studies by Mathews, Ridgeway, and Holmes (2013) confirm that mental images are perceived as more realistic and involve stronger emotions than verbal representations.

Because of the similarity to percepts, mental images can also cause direct emotional reactions and bypass higher cognitive processes (including language processing) - as is often the case with emotional perceptual stimuli. This is evident in posttraumatic stress disorders, where without the participation of higher cognitive functions, patients manifest automatic emotional, physiological, and behavioral reactions in response to traumatic intrusive images (Burnett Heyes et al., 2013; Holmes \& Mathews, 2010). 
The third explanation of the relation between emotions and mental images that was suggested by Holmes and Mathews (2010) concerns the mediating role of memory processes. The authors point out that brain areas that are active while imagining something also overlap with areas associated with the recall of autobiographical memories. It is possible that the creation of mental images - regardless of whether they depict events from the past, the future, or are completely unrealistic - is achieved by combining fragments of our own memories. As a result, the emotions attributed to the memories used in this process are excited. To confirm this mechanism of mental image creation, Holmes and Mathews (2010) refer, among others, to a study of people with amnesia who, having lost the ability to recall memories of events from before their brain injury, were also unable to create new mental images concerning themselves.

The authors emphasize that in the theory of Conway and Pleydell-Pearce (2000), emotional autobiographical memories are stored just with image-like representations. This is in line with research showing that the emotional characteristics of the presented story affect memory of perceptual aspects of the material more than they affect the memory of its conceptual or verbal aspects (Arntz, De Groot, \& Kindt, 2005). Conway (2001) in his conception of episodic memory describes memory traces that serve to monitor progress in the fulfilling of a person's goals, as mainly characterized by "sensory-perceptual" information. In this way, the relationship between memories, emotions, and mental images appears again, as approaching or moving away from a goal evokes emotions of a certain type. Holmes and Mathews (2010), who gathered data from research on image representations and in the context of the three presented mechanisms, created a heuristic model showing the greater impact of image representations on emotional processes compared to verbal ones. The model also shows the greater effect of images on readiness to act, beliefs about the realness of presented content, and the assessment of oneself and one's image.

The specificity of mental images can make them a source of discomfort and difficulties in some mental disorders. Perhaps people suffering from these disorders need a specially developed strategy to suppress harmful thoughts. It has been proven that providing a suppression strategy helps in eliminating unwanted thoughts in older people who, without such a strategy, deal with suppression worse than younger adults (Murray, Anderson, \& Kensinger, 2015). Similarly, creating appropriate conditions allows the elimination of individual differences related to distraction sensitivity, which can be understood as a deeper processing of content that is unrelated to the current goal, including intrusions (Forster \& Lavie, 2007). On the other hand, people with obsessive-compulsive disorder use not only ineffective but even harmful methods of dealing with intrusions, such as punishing themselves for the appearance of unwanted thoughts (Abramowitz, Whiteside, Kalsy, \& Tolin, 2003; Amir, Cashman, \& Foa, 1997). Healthy people may also experience distress caused by thinking with images; according to estimates, as much as $80 \%$ of the healthy population struggles with intrusions (Rachman \& de Silva, 1978). Therefore, there is a great need for effective and healthy strategies for the elimination of the harmful consequences of intrusive images or for the regulation of emerging thoughts. 
The effects of thinking with images depend, however, on the content of the images. If we want to get rid of harmful image thoughts, it is worth considering using other images for this purpose that have similar characteristics that are related to the format of representation, but with friendlier content. For a long time, clinicians have used mental image interventions, among others, in the treatment of post-traumatic stress disorders (Brewin et al., 2010). Using image representations to suppress thoughts seems justified because these representations, due to their realistic character and special relationship with emotions, have the potential to absorb attention and motivate a person to sustain them in their consciousness. In addition, volitional thinking with images should fully employ the cognitive resources needed by intrusive images to become conscious.

In the next part of the article, I will try to find a strategy that, thanks to the use of images, could help to suppress unwanted thoughts. To this end, I will describe reports from three research areas: research in two paradigms of thought suppression (the "think/no-think" paradigm and the "white bear" paradigm) and research on the effects of the perceptual and cognitive control load on the processing of target-unrelated stimuli (also intrusions). Based on these works, I will draft a strategy for dealing with unwanted thoughts using image representations. Next, I will review the research in a way that verifies the validity of the proposed strategy.

\section{Factors Influencing Thought Suppression: The "Think/No-Think" Paradigm}

Two general mechanisms which have the potential to help in getting rid of unwanted mental contents from consciousness can be indicated: 1) engaging the cognitive resources needed to process intrusions; 2) reducing the availability of the intrusions (or blocking access to them). There are also two theoretical and research approaches to thought suppression, each of which emphasizes a different mechanism. These approaches are related to two paradigms of thought suppression: the "think/no-think" paradigm and the "white bear" paradigm.

Research conducted around the "think/no-think" paradigm indicates that getting rid of a thought is possible due to the processes that reduce that thought's availability (Anderson \& Green, 2001). In research in this paradigm, participants firstly learn word pairs, then they are shown the first words (cue words) of most of the pairs. For some pairs, participants are presented with a cue word and have to recall the second word of the pair (the target word), and with other pairs they have to suppress thought of the second word. The more often a participant is presented with the cue word for a target word they are told not to think of, the more difficult it is for them to recall this word in the subsequent memory test. Suppressed words are usually remembered worse not only than words that should have been recalled when participants were presented with their cues, but also than the words that had been learnt in the previous part of the experiment but whose cues did not appear in the second phase (baseline words). In other words, the suppressed contents, despite the presentation of their cues, are remembered worse than contents that the participants were not reminded of. 
This so-called negative control effect persists even when participants receive money for each correctly recalled word, while it disappears when instead of suppression there is an instruction to refrain from saying the word (Anderson \& Green, 2001). Neuroimaging studies have shown that during the presentation of cues of suppressed contents, the activity of the hippocampus (the structure responsible, among others, for conscious recollection) is lower than when looking at the fixation items that acted as the baseline (Depue, Curran, \& Banich, 2007). However, the reduction in the activity of this structure occurs through the prefrontal areas that are responsible for cognitive control (Anderson et al., 2004). All these results indicate that it is possible to volitionally reduce the availability of selected mental content.

The negative control effect was repeatedly replicated. It was visible in the results of research carried out on a group of nearly seven hundred people in total (Levy \& Anderson, 2008). At the same time, there are individual differences regarding the size of the effect. The metaanalysis showed that suppressed words are recalled on average $6 \%$ worse than baseline words, but in some people this effect size reaches $60 \%$; in others, the effect does not appear at all, but there is better (up to $40 \%$ ) remembering of suppressed words than baseline words.

According to Levy and Anderson (2008), these differences are caused by individual differences in cognitive control abilities. The authors describe research which shows that people with greater working memory capacity (which is associated with cognitive control capabilities) have a greater negative control effect as well. The relationship between cognitive control and thought suppression is also confirmed by research involving participants in various age groups. It is believed that cognitive control abilities are lower in the elderly and children compared to younger adults; children and the elderly suppress less well in the "think/no-think" procedure (Anderson, Reinholz, Kuhl, \& Mayr, 2011; Paz-Alonso, Ghetti, Matlen, Anderson, \& Bunge, 2009). However, the ability to suppress thoughts increases with children's age (Paz-Alonso et al., 2009).

From research in the "think/no-think" paradigm, one can conclude that the success of thought suppression depends on cognitive control capabilities. There are individual differences regarding cognitive control. People with better control should usually suppress better, but this does not mean that only people in this group can regulate their own thoughts. The effectiveness of thought suppression probably also depends on how they are suppressed or the conditions in which the suppression takes place. For example, as already mentioned, when older people are provided with a proper strategy, they suppress thoughts just as well as younger adults, but without such a strategy they perform worse (Murray et al., 2015).

One can imagine conditions in which even people with enormous potential for cognitive control would not be able to use it to block intrusions. For example, when a person is engaged in several tasks involving executive functions, there might be no resources left to conduct successful thought suppression. However, there might also be conditions with free access to cognitive control resources. Such circumstances should especially favor people with less cognitive control abilities, for which access to relatively few resources can determine the success or failure of thought suppression. In conclusion, the chance of effective thought suppression should increase under conditions of free access to cognitive control resources. 


\section{Factors Influencing Thought Suppression: The "White Bear" Paradigm}

In research around the "white bear" paradigm (Wegner, Schneider, Carter, \& White, 1987), it is also seen that the success of suppression depends on certain mental resources. Participants suppress chosen mental content in experiments in this paradigm; for example, the thought of a white bear. How often the forbidden thought appears during and after suppression is measured introspectively, and the results are compared to the results of the control group that did not suppress thoughts. Often replicated was the paradoxical rebound effect, which involves more unwanted thoughts after suppression than when there were no suppression attempts (for review, see: Abramowitz, Tolin, \& Street, 2001; Rassin, 2005; Wenzlaff \& Wegner, 2000).

There are studies that even seem to hold evidence of the hyperaccessibility of the suppressed content. In one of these studies (Wegner \& Erber, 1992, experiment 2), some participants suppressed thoughts in a high cognitive load condition that involved maintaining a multi-digit number in memory. During suppression (or during concentration in the control group), participants performed a Stroop task in which the target content also appeared. The reaction time to the suppressed content was slower under high than under low cognitive load. In the high cognitive load condition, the reaction time to the target content was also marginally significantly slower in the suppression group than in the concentration group. The slower reaction time in the Stroop task was interpreted as an indicator of the higher accessibility of the content.

The results observed in these studies are explained by Wegner's theory of ironic processes of mental control (1994), according to which thought suppression requires two processes: the operating process, which searches for contents that redirect attention from the unwanted content, and the monitoring process, which is sensitive to the appearance of intrusions. The conscious operating process looks for alternative material and fills the consciousness with it, thus blocking conscious access to the unwanted content. The unconscious monitoring process, however, assesses the risk of unwanted content appearing in the consciousness despite the intention to suppress it.

The operating process is effortful and does not work all the time. If the monitoring process detects an unwanted thought, the operating process resumes its work. The theory assumes that thoughts can be suppressed with some success if there are enough resources to supply the operating process. If these resources are lacking, paradoxical effects will appear in the form of hyperaccessibility of the suppressed content. This is a side effect of the monitoring process, which while it is sensitive to unwanted thought, at the same time increases its activation. If the activation of the suppressed thought is not balanced by the operating process, which increases the availability of competing alternative thoughts, a person begins to think about the unwanted content more often than if they did not suppress it at all.

In light of the theory and research related to the "white bear" paradigm described above, it seems reasonable to draw two conclusions: First, it is necessary for thought suppression to 
fill consciousness with content other than that which is avoided. Second, suppression success depends on the availability of the resources that supply the operating process.

The second conclusion is somewhat in line with the conclusions of research in the "think/no-think" paradigm, for which resources related to cognitive control or executive functions play a crucial role. It seems that the description given above of the operating process fits well in the definition of executive functions as functions that are involved in control of cognitive processes in accordance with a specific goal (Nigg, 2017). The difference between the conclusions from the research in both paradigms is that in the "think/nothink" paradigm the resources associated with executive functions allowed success in thought suppression, while in the "white bear" paradigm they mainly prevent paradoxes.

The reason for these differences is still unclear. The difficulty is also related to the fact that the very issue of executive functions and associated resources is insufficiently described. There are a number of distinguished executive functions, with research indicating their "unity and diversity," which means they are partly related to and partly independent of each other (Friedman \& Miyake, 2017). Inhibition is one of the explored functions. As with research in the "think/no-think" paradigm, we have premises that participants inhibit the activation of the suppressed content, but in experiments in the "white bear" paradigm there is no sign of this. Why this is so remains unanswered. Similarly, it is not known whether different executive functions use the same resource pool or whether each type of function draws from its separate pool.

Nevertheless, in trying to combine the conclusions of both research areas it seems that the probability of the success of thought suppression could be increased by a strategy in which a person would be provided with alternative content that distracts their attention from unwanted thoughts while at the same time freeing up resources for executive functions. When we add to this what we know about the properties of image representations, i.e. their special impact on emotions, we can suggest that alternative content should take the form of an image. Such a strategy seems all the more promising as it corresponds with the theory and research of Lavie and colleagues that is related to the different impacts of perceptual and cognitive control load on the susceptibility to distraction, including intrusions.

\section{Factors Influencing Thought Suppression: Lavie's Attention Theory}

Lavie and colleagues (Lavie, 2010; Lavie, Hirst, De Fockert, \& Viding, 2004) noticed that in a task requiring an adequate response to a specific perceptual stimulus, the susceptibility to interference induced by distractors depends on the type of the cognitive load. In the case of high perceptual load (e.g. an increase in the number or complexity of the stimuli among which an important stimulus must be sought), the interference is smaller than when the load is low. This is explained by the fact that the resources associated with perceptual processing at high perceptual load are exhausted and are not sufficient for deeper processing of distractors. On the other hand, under low load all visible stimuli, including distractors, are automatically processed more deeply. 
It is the opposite situation in the case of cognitive control load: at higher load, interference caused by distractors increases, while at lower load it decreases. Cognitive control is responsible for matching responses to current goals. For example, when the perceptual load is low and deeper distraction processing occurs, the control processes prevent the impulsive response to the distractor and promote a response that is adequate to task requirements. However, when cognitive control resources are occupied (for example, by the necessity of maintaining complex material in working memory), the interference induced by the distraction increases, therefore it is more difficult to generate the correct reactions. In conclusion, high perceptual load reduces the susceptibility to distraction, and high cognitive control load increases it.

The theory of Lavie and co-workers (2004) shows that the involvement of resources associated with processing of important perceptual stimuli prevents deeper processing of distracting, disturbing, or unwanted stimuli, which thus cannot reach consciousness. It can be argued that the loading of perceptual resources with selected content will hinder intrusion processing. This prediction gained partial support in studies showing that under higher perceptual load, not only external distractors but also internal distractors are processed more poorly (Forster \& Lavie, 2009). The results indicate that with perceptual load there are fewer thoughts unrelated to the performed task, including — as is essential — unwanted thoughts. This prediction is also in line with the theory of ironic processes of mental control, assuming the need to fill consciousness with alternative content in order to divert attention away from unwanted thoughts. Wegner (1994), however, did not specify whether the alternative content should take the form of an image or of a representation of a different kind. Lavie points to perceptual load, and therefore to image representations.

On the other hand, Lavie's theory predicts that the lack of cognitive control resources may cause the unwanted content to have more impact on a person. This seems consistent with Wegner's theory and conclusions from research in the "think/no-think" paradigm. Here, in the case of insufficient cognitive control or executive function resources, unwanted content appears more often, is more strongly activated, and is better remembered.

\section{Suppressing with Images: the Proposition of Thought Suppression Strategy}

Research around the "think/no-think" paradigm seems to indicate that thought suppression depends on the possibility of using executive functions. The freeing up of the resources that are required by executive functions to work well should promote effective suppression, while occupying them should hinder it. Research related to the "white bear" paradigm also proves that loading cognitive control resources counteracts suppression. In contrast, thought suppression is supported by thinking about something other than the unwanted content. All this is consistent with the theory of Lavie and co-workers (2004), in which the perceptual load deprives a person of the resources needed for deeper processing of unwanted external and internal stimuli, and cognitive control load reduces resistance to distraction interference. 
From these conclusions, we can draw some recommendations regarding the strategy of thought suppression. This strategy consists of redirecting attention through certain images while leaving cognitive control resources maximally freed up.

The use of images to fight intrusions is argued by the research and theory of Lavie and colleagues (2004), in which perceptual load was the one to hinder processing of unwanted contents - both external and internal (Forster \& Lavie, 2009). Also, the previously described properties of image representations make them a promising way of supporting thought suppression.

Image representations evoke emotions more easily than verbal representations. This means that a person could be more motivated to keep images in consciousness and to develop imagined scenarios. For example, imagining a nice meeting should more strongly stimulate pleasant emotions and the desire to fantasize about its course than processing information about the meeting in a way that does not affect imagination. An image strengthened by emotions and motivation becomes a stronger rival in the competition with intrusions for access to consciousness.

Intrusive content is often associated with unpleasant emotions, including excessive anxiety or even terror in response to a threat of failure in achieving an important goal. This happens, for example, in the case of the intrusions that accompany obsessive-compulsive disorders when a person is worried about their health, or in social phobia when intrusions represent a threat of public humiliation. In these examples, the understanding of the situation is mostly inadequate as the likelihood of a threat is overestimated. It might be possible to counteract this by using image representations, which seem more real than verbal representations. The representation affects emotions, behavior, and beliefs about the degree of realization of the goal it refers to. When imagining a safe situation or one in which an important goal is pursued, one can weaken a belief about a threat that does not exist in reality.

Finally, volitionally chosen images should be particularly useful in fighting intrusive images. Since images so successfully arouse emotions, intrusive images must have extremely severe repercussions. Imagining or perceiving something takes the limited resources of the so-called visuo-spatial sketchpad, which is responsible for maintaining image representations in working memory (Baddeley, 2012). A volitionally chosen pleasant image representation can take the resources of the sketchpad and thus prevent painful intrusions being stored there. This does not mean that images can be only used against other images. According to Oberauer (2009), there can be only one mental content in consciousness at any one time. Conscious processing of an image should block access to awareness of other contents - be they images or other representations. The occupation of the visuo-spatial sketchpad (in Oberauer's theory, this would be an area of direct access, i.e. the area of content that can be made conscious in the near future) further reduces the probability of intrusive images appearing in the consciousness. 
For these reasons, the proposed strategy assumes the use of images to counter intrusions; this should take place in conditions that are least likely to load cognitive control resources with tasks other than the task of thought suppression. The question arises as to what exactly such conditions would be.

Unfortunately, it is difficult to provide a precise answer. As mentioned earlier, there are many executive functions. It is not known whether all functions use one or many resource pools. When speaking of cognitive control, Lavie (2010) means actively maintaining current goals related to the performed task or switching between tasks, both of which are examples of executive functions; other examples are updating working memory and inhibition. It is safe to assume that if we want to suppress thoughts, it may be better not to simultaneously carry out tasks that involve these processes. There is a risk that such tasks will interfere with possible inhibition of unwanted content, maintaining the active goal of avoiding that content, and filling working memory with alternative content.

\section{The Thought Suppression Strategy in the Light of Existing Empirical Data}

Of course, the proposed strategy has not been directly experimentally verified; however, there are some studies that may shed light on the validity of the assumptions.

There are studies confirming that focusing on a mental image helps to suppress thoughts. In the study of Lin and Wicker (2007), creating a mental picture of a kitchen contributed to a reduction of the number of intrusions associated with the previously presented highly unpleasant story of a traffic accident and to a reduction of the level of anxiety compared to conditions without the mental picture. Similarly, in Luciano and González (2007), imagining a place unrelated to a terrorist attack proved to be the most effective way of reducing the number of intrusions related to a specific terrorist event. However, in the comparison groups of these studies the participants did not receive an instruction to replace unwanted thoughts with a selected verbal representation; instead, they were asked to suppress their thoughts without specifying how this could be done.

Studies in the "think/no-think" paradigm also indicate that substituting a suppressed thought with another one usually helps in suppression (Hertel \& Calcaterra, 2005; for review, see Anderson \& Huddleston, 2012). In a study with depression sufferers, only the strategy of substituting negative words with positive ones allowed effective suppression (Joormann, Hertel, LeMoult, \& Gotlib, 2009). Here, however, no images were used; the alternative contents were words.

The effectiveness of using image and non-image representations to fight unwanted thoughts was explored by Harvey and Payne (2002). In this experiment, participants were people with sleep problems caused by troublesome thoughts appearing before falling asleep. Participants who imagined something interesting, engaging, pleasurable, and relaxing in order to distract attention from unwanted thoughts fell asleep faster than participants who diverted their attention without using imagination and participants from the 
control group. In the group using imagination, appearing intrusions were associated with the relatively least discomfort (however, there were no differences in the amount of these thoughts). Only people from the imagination group estimated that they fell asleep faster during the experiment than usual.

Thinking with images in order to suppress unwanted thoughts, however, does not necessarily mean thinking with visual images or imagining a stimulus without its real presence. Also, focusing on the sensory data available at a given moment may prove helpful. Research by Ju and Lien (2016) suggests that such images may be more supportive to thought suppression as they involve less cognitive control or working memory than imagining something. The authors compared the effectiveness of two strategies that accompany thought suppression: maintaining a mental image (of a blue sports car) and focusing on one's own breath. Participants concentrating on breath-related experiences had fewer intrusions during suppression than people from the imagination group. In addition, the number of intrusions in the group that concentrated on breath did not correlate with the capacity of working memory (i.e. a certain indicator of the cognitive control capability), while there was a negative correlation between the latter measure and the number of intrusions in the imagination group. The authors indicate that concentration on breath may require less cognitive control: here it is enough that the person directs attention to the current experience, which is more easily accessible than imagination, which needs to be created and maintained. This might have led to more cognitive control resources being delegated to thought suppression. The independence of the success of the strategy of focusing on breath from the resources of cognitive control means that even people with lower abilities to control their thoughts have a chance to effectively suppress them if provided with a proper strategy.

In their study, Salkovskis and Reynolds (1994) also used focusing on breath in order to suppress smoking-related intrusions in nicotine addicts. There was a decrease in frequency of intrusions despite the requirement to control the breath in some way as this probably depends on cognitive control resources, although it seems that due to the low level of task difficulty, the load was not very high. Similarly, an intervention of body scanning involving focusing on body sensations had a favorable effect on eliminating smoke-related thoughts and reducing cigarette cravings (May, Andrade, Willoughby, \& Brown, 2012). Cigarette cravings were also reduced more efficiently by imaging a beach in comparison to counting down in intervals of seven (Versland \& Rosenberg, 2007). It is believed that imagination can both amplify and attenuate cravings (Andrade, May, \& Kavanagh, 2012).

Besides the use of images, the proposed thought-suppressing strategy involves freeing up as many cognitive control resources as possible. This is supported by research which shows that cognitive load that involves maintaining easy-to-forget material (e.g. a multidigit number) in working memory impairs thought suppression (e.g. Macrae, Milne, Bodenhausen, \& Ford, 1997; Nixon, Cain, Nehmy, \& Seymour, 2009; Nixon \& Rackebrandt, 2016; Wegner \& Erber, 1992; Wegner, Erber, \& Zanakos, 1993; Wenzlaff \& Bates, 2000). 
According to Wegner (1994), this is because the operating process - filling consciousness with content that is not avoided - cannot work because of resource deficiency. However, is maintaining a multi-digit number in consciousness not an activity that is carried out precisely by this type of process? If it is, then cognitive control resources would be absorbed just by the operating process.

In my opinion, the problem with thought suppression in these studies is related to the fact that tasks that load cognitive control are too demanding. It is not so much about lacking resources to supply the operating process as about the fact that this process is not able to perform such difficult tasks. Constant focusing on arbitrarily chosen, meaningless, and uninteresting material exhausts the resources, making the task difficult to continue. At the moment when control relaxes, intrusions may pop into consciousness. Their emergence is all the more likely when the alternative content is not related to any important personal goals. At least some of the intrusions, however, are closely related to individual goals and the threat of not fulfilling them. Thus, there arises a situation in which it is difficult to focus on meaningless content and there is a sense of a threat to some important goal. In this context, the appearance of intrusions seems justified because it may be a result of a mechanism that directs our actions to the fulfilment of goals.

However, when intrusions are clearly undesirable or harmful, it seems more appropriate to block their access to consciousness by thinking of contents that are relatively easy to think of. Such a task does not overload cognitive control, so it can be accomplished more effectively and serve as a better thought suppression support. This is confirmed by the previously mentioned study of Ju and Lien (2016), in which better thought suppression effects were achieved by focusing on the breath, which is available at any time, than on a mental image that has to be constantly maintained or re-actualized in working memory.

In addition, the absorption of cognitive control resources by a demanding task could make it harder to simultaneously inhibit the accessibility of unwanted content. In other words, firstly there is a difficulty in maintaining the alternative content, and secondly there may be not enough resources to reduce the availability of the unwanted content. This assumption is confirmed by research in the "think/no-think" paradigm, in which people under higher working memory load more often recalled suppressed words as an association with a new cue than people without the load (Noreen \& De Fockert, 2017). It is not known how thought suppression is influenced by a demanding but exciting task. Is it possible to inhibit unwanted content under such conditions? It may depend on the kind of executive functions used in the task, but this is a problem that requires further exploration.

There are studies that at first glance seem to contradict the proposed strategy because they support the hypothesis that a load on cognitive control processes supports thought suppression. For example, Teasdale and colleagues (1995) suggest that stimulus-unrelated thoughts, including intrusions, could be prevented by the engagement of the so-called central executive, i.e. a construct that in Baddeley's working memory theory (2012) is responsible for a number of control functions. In the study, three components of working memory were loaded: the central executive, which is responsible for control and coordination of 
ongoing processes; the phonological loop, which stores currently needed contents encoded in phonological code (such as spoken words); and the visuo-spatial sketchpad, which stores currently needed mental images (experiments 1 and 2). The experimental manipulation helped to reduce the frequency of stimulus-independent thoughts as compared to the control conditions without the load. There were no differences in the number of these thoughts between conditions of central executive load and storage systems load. However, because the task that loaded the phonological loop and the visuo-spatial sketchpad involved (although to a lesser extent) the central executive, the authors hypothesized that the last system is crucial to the decrease in stimulus-unrelated thoughts. This was confirmed by further experiments in which there was a lower frequency of stimulus-unrelated thoughts in a less automated task (experiment 3) and in random generation of digits (experiment 4, where randomness was considered a manifestation of the involvement of the central executive).

How can these results be reconciled with previously cited studies? Perhaps Teasdale and colleagues' results (1995) are related not so much to a greater involvement of cognitive control as to a more frequent filling of consciousness with task-related stimuli. After all, there were no differences in the effectiveness of reducing the frequency of stimulus-unrelated thoughts between experimental conditions (experiments 1 and 2). Moreover, (in reference to experiments 3 and 4) automatic activities tend to involve not only less control but also less consciousness than non-automatic activities (Moors \& De Houwer, 2006). This hypothesis finds some support in the research of Teasdale, Proctor, Lloyd and Baddeley (1993; although not in their interpretation of the outcomes). The results show that when stimuli are presented rapidly, sustaining them in the memory and simply repeating them equally contributes to reducing the frequency of stimulus-unrelated thoughts as compared to control conditions. With a slow presentation rate, repetition was less effective. In this case, there were longer periods when attention and consciousness were not directed to the task-related stimulus. Similarly, the memory task less effectively reduced the number of thoughts unrelated to the task when the awareness of the stimuli kept in the memory was lower. Hence, it could be argued that it is not the central executive load that helps in reducing the number of unwanted thoughts but the frequency of filling the consciousness with stimuli associated with the task (whether as a result of top-down or bottom-up processes).

\section{Conclusions}

In the article, I proposed a thought suppression strategy which involves diverting attention from unwanted thoughts by thinking with volitionally chosen images without the accompanying cognitive control load.

The use of images is argued due to their special ability to evoke emotions. Research indicates that image representations can stimulate emotions more strongly and their content is perceived as more realistic in comparison to verbal representations. On the one hand, this makes images stronger rivals in the competition for access to consciousness. On the other hand, processing images takes resources that otherwise could be used to process intrusive 
images which may be particularly harmful due to the aforementioned special relationship between images and emotions. Also, it may be useful to note that the supportive value of an alternative image may depend on its content. As intrusions often alert that a person's goal is under (unrealistic) threat, maybe it would be helpful to suppress these intrusions using images that illustrate goal fulfilment. This idea has some support in the latest empirical data (Wang, Chatzisarantis, \& Hagger, 2017, 2018).

The absence of cognitive control load should in turn enable the directing of all possible resources to carry out the thought suppression task. Thought suppression is most likely done by cognitive control, i.e. by executive functions like inhibition, working memory updating, or maintaining goal representation.

The strategy seems reasonable in light of Lavie's theory of attention and research in the "think/no-think" and the "white bear" paradigms, as well as other studies on thought suppression. However, it has not been verified directly. Still, it should be remembered that the strategy would probably not apply to all cases. For example, sometimes intrusions are so invasive that it may be hard to redirect attention away from them. In that case, other methods may prove helpful, such as a method involving specific eye movements during an experience of visual intrusions that seems to attenuate emotions that are evoked by them (van Den Hout, Muris, Salemink, \& Kindt, 2001); after this intervention, the proposed strategy might be used. Nevertheless, there is firstly a need to conduct experiments that directly test if and maybe when and how exactly the strategy works.

\section{References}

Abramowitz, J. S., Whiteside, S., Kalsy, S. A., \& Tolin, D. F. (2003). Thought control strategies in obsessive-compulsive disorder: A replication and extension. Behaviour Research and Therapy, 41(5), 529-540. https://doi.org/10.1016/S0005-7967(02)00026-8

Abramowitz, Jonathan S., Tolin, D. F., \& Street, G. P. (2001). Paradoxical effects of thought suppression: A meta-analysis of controlled studies. Clinical Psychology Review, 21(5), 683-703. https://doi.org/10.1016/S0272-7358(00)00057-X

Amir, N., Cashman, L., \& Foa, E. B. (1997). Strategies of thought control in obsessive-compulsive disorder. Behaviour Research and Therapy, 35(8), 775-777. https://doi.org/10.1016/S00057967(97)00030-2

Anderson, M. C., \& Green, C. (2001). Suppressing unwanted memories by executive control. Nature, 2001(410), 366-369. https://doi.org/10.1038/35066572

Anderson, Michael C., \& Huddleston, E. (2012). Towards a cognitive and neurobiological model of motivated forgetting. In R. F. Belli (Ed.), Nebraska symposium on motivation: Vol. 58. True and false recovered memories: Toward a reconciliation of the debate (pp. 53-120). New York, NY, US: Springer Science + Business Media. https://doi.org/10.1007/978-1-4614-1195-6_3 
Anderson, M. C., Ochsner, K. N., Kuhl, B., Cooper, J., Robertson, E., Gabrieli, S. W., Glover, G. H., \& Gabrieli, J. D. (2004). Neural systems underlying the suppression of unwanted memories. Science, 303(5655), 232-235. https://doi.org/10.1126/science.1089504

Anderson, Michael C., Reinholz, J., Kuhl, B. A., \& Mayr, U. (2011). Intentional suppression of unwanted memories grows more difficult as we age. Psychology and Aging, 26(2), 397-405. https://doi.org/10.1037/a0022505

Andrade, J., May, J., \& Kavanagh, D. (2012). Sensory imagery in craving: From cognitive psychology to new treatments for addiction. Journal of Experimental Psychopathology, 3(2), 127-145. https://doi.org/10.5127/jep.024611

Arntz, A., De Groot, C., \& Kindt, M. (2005). Emotional memory is perceptual. Journal of Behavior Therapy and Experimental Psychiatry, 36(1), 19-34.

https://doi.org/10.1016/j.jbtep.2004.11.003

Baddeley, A. (2012). Working memory: Theories, models, and controversies. Annual Review of Psychology, 63(1), 1-29. https://doi.org/10.1146/annurev-psych-120710-100422

Blackwell, S. E., Rius-Ottenheim, N., Schulte-van Maaren, Y. W., Carlier, I. V., Middelkoop, V. D., Zitman, F. G., Spinhoven, P., Holmes, E. A., \& Giltay, E. J. (2013). Optimism and mental imagery: A possible cognitive marker to promote well-being? Psychiatry Research, 206(1), 56-61. https://doi.org/10.1016/j.psychres.2012.09.047

Brewin, C. R., Gregory, J. D., Lipton, M., \& Burgess, N. (2010). Intrusive images in psychological disorders: characteristics, neural mechanisms, and treatment implications. Psychological Review, 117(1), 210-232. https://doi.org/10.1037/a0018113

Burnett Heyes, S., Lau, J. Y. F., \& Holmes, E. A. (2013). Mental imagery, emotion and psychopathology across child and adolescent development. Developmental Cognitive Neuroscience, 5, 119-133. https://doi.org/10.1016/j.den.2013.02.004

Çili, S., \& Stopa, L. (2015). Intrusive mental imagery in psychological disorders: Is the self the key to understanding maintenance? Frontiers in Psychiatry, 6, 103-107. https://doi.org/10.3389/fpsyt.2015.00103

Conway, M. A. (2001). Sensory-perceptual episodic memory and its context: Autobiographical memory. Philosophical Transactions of the Royal Society B: Biological Sciences, 356(1413), 1375-1384. https://doi.org/10.1098/rstb.2001.0940

Conway, M. A., \& Pleydell-Pearce, C. W. (2000). The construction of autobiographical memories in the self-memory system. Psychological Review, 107(2), 261-288.

https://doi.org/10.1037//0033-295X.107.2.261

Depue, B. E., Curran, T., \& Banich, M. T. (2007). Prefrontal regions orchestrate suppression of emotional memories via a two-phase process. Science, 317(5835), 215-219.

https://doi.org/10.1126/science. 1139560

Forster, S., \& Lavie, N. (2007). High perceptual load makes everybody equal. Psychological Science, 18(5), 377-381. https://doi.org/10.1111/j.1467-9280.2007.01908.x

Forster, S., \& Lavie, N. (2009). Harnessing the wandering mind: The role of perceptual load. Cognition, 111(3), 345-355. https://doi.org/10.1016/j.cognition.2009.02.006 
Friedman, N. P., \& Miyake, A. (2017). Unity and diversity of executive functions: Individual differences as a window on cognitive structure [Special issue]. Cortex, 86, 186-204. https://doi.org/10.1016/j.cortex.2016.04.023

Harvey, A. G., \& Payne, S. (2002). The management of unwanted pre-sleep thoughts in insomnia: Distraction with imagery versus general distraction. Behaviour Research and Therapy, 40(3), 267-277. https://doi.org/10.1016/S0005-7967(01)00012-2

Hertel, P. T., \& Calcaterra, G. (2005). Intentional forgetting benefits from thought substitution. Psychonomic Bulletin and Review, 12(3), 484-489. https://doi.org/10.3758/BF03193792

Holmes, E. A., Lang, T. J., \& Shah, D. M. (2009). Developing interpretation bias modification as a "cognitive vaccine" for depressed mood: Imagining positive events makes you feel better than thinking about them verbally. Journal of Abnormal Psychology, 118(1), 76-88. https://doi.org/10.1037/a0012590

Holmes, E. A., \& Mathews, A. (2005). Mental imagery and emotion: A special relationship? Emotion, 5(4), 489-497. https://doi.org/10.1037/1528-3542.5.4.489

Holmes, E. A., \& Mathews, A. (2010). Mental imagery in emotion and emotional disorders. Clinical Psychology Review, 30(3), 349-362. https://doi.org/10.1016/j.cpr.2010.01.001

Holmes, E. A., Mathews, A., Dalgleish, T., \& Mackintosh, B. (2006). Positive interpretation training: Effects of mental imagery versus verbal training on positive mood. Behavior Therapy, 37(3), 237-247. https://doi.org/10.1016/j.beth.2006.02.002

Holmes, E. A., Mathews, A., Mackintosh, B., \& Dalgleish, T. (2008). The causal effect of mental imagery on emotion assessed using picture-word cues. Emotion, 8(3), 395-409. https://doi.org/10.1037/1528-3542.8.3.395

Joormann, J., Hertel, P. T., LeMoult, J., \& Gotlib, I. H. (2009). Training forgetting of negative material in depression. Journal of Abnormal Psychology, 118(1), 34-43. https://doi.org/10.1037/a0013794

Ju, Y. J., \& Lien, Y. W. (2016). Better control with less effort: The advantage of using focusedbreathing strategy over focused-distraction strategy on thought suppression. Consciousness and Cognition, 40, 9-16. https://doi.org/10.1016/j.concog.2015.12.002

Lavie, N. (2010). Attention, distraction, and cognitive control under load. Current Directions in Psychological Science, 19(3), 143-148. https://doi.org/10.1177/0963721410370295

Lavie, N., Hirst, A., De Fockert, J. W., \& Viding, E. (2004). Load theory of selective attention and cognitive control. Journal of Experimental Psychology: General, 133(3), 339-354. https://doi.org/10.1037/0096-3445.133.3.339

Levy, B. J., \& Anderson, M. C. (2008). Individual differences in the suppression of unwanted memories: The executive deficit hypothesis. Acta Psychologica, 127(3), 623-635. https://doi.org/10.1016/j.actpsy.2007.12.004

Lin, Y. J., \& Wicker, F. W. (2007). A comparison of the effects of thought suppression, distraction and concentration. Behaviour Research and Therapy, 45(12), 2924-2937. https://doi.org/10.1016/j.brat.2007.08.004 
Luciano, J. V., \& González, S. A. (2007). Analysis of the efficacy of different thought suppression strategies. International Journal of Psychology and Psychological Therapy, 7(3), 335-345.

Macrae, C. N., Milne, A. B., Bodenhausen, G. V., \& Ford, R. L. (1997). On the regulation of recollection: The intentional forgetting of stereotypical memories. Journal of Personality and Social Psychology, 72(4), 709-719. https://doi.org/10.1037/0022-3514.72.4.709

Mathews, A., Ridgeway, V., \& Holmes, E. A. (2013). Feels like the real thing: Imagery is both more realistic and emotional than verbal thought. Cognition and Emotion, 27(2), 217-229. https://doi.org/10.1080/02699931.2012.698252

May, J., Andrade, J., Willoughby, K., \& Brown, C. (2012). An attentional control task reduces intrusive thoughts about smoking. Nicotine and Tobacco Research, 14(4), 472-478. https://doi.org/10.1093/ntr/ntr238

Miller, G. A., Levin, D. N., Kozak, M. J., Edwin, E., McLean, A., \& Lang, P. J. (1987). Individual differences in imagery and the psychophysiology of emotion. Cognition and Emotion, 1(4), 367-390. https://doi.org/10.1080/02699938708408058

Moors, A., \& De Houwer, J. (2006). Automaticity: A theoretical and conceptual analysis. Psychological Bulletin, 132(2), 297-326. https://doi.org/10.1037/0033-2909.132.2.297

Murray, B. D., Anderson, M. C., \& Kensinger, E. A. (2015). Older adults can suppress unwanted memories when given an appropriate strategy. Psychology and Aging, 30(1), 9-25. https://doi.org/10.1037/a0038611

Nigg, J. T. (2017). Annual Research Review: On the relations among self-regulation, self-control, executive functioning, effortful control, cognitive control, impulsivity, risk-taking, and inhibition for developmental psychopathology. Journal of Child Psychology and Psychiatry and Allied Disciplines, 58(4), 361-383. https://doi.org/10.1111/jcpp.12675

Nixon, R. D. V., Cain, N., Nehmy, T., \& Seymour, M. (2009). The influence of thought suppression and cognitive load on intrusions and memory processes following an analogue stressor. Behavior Therapy, 40(4), 368-379. https://doi.org/10.1016/j.beth.2008.10.004

Nixon, R. D. V., \& Rackebrandt, J. (2016). Cognitive load undermines thought suppression in acute stress disorder. Behavior Therapy, 47(3), 388-403. https://doi.org/10.1016/j.beth.2016.02.010

Noreen, S., \& De Fockert, J. W. (2017). The role of cognitive load in intentional forgetting using the think/no-think task. Experimental Psychology, 64(1), 14-26. https://doi.org/10.1027/1618$3169 / \mathrm{a} 000347$

Oberauer, K. (2009). Design for a working memory. Psychology of Learning and Motivation, 51, 45-100. https://doi.org/10.1016/S0079-7421(09)51002-X

Paz-Alonso, P. M., Ghetti, S., Matlen, B. J., Anderson, M. C., \& Bunge, S. A. (2009). Memory suppression is an active process that improves over childhood. Frontiers in Human Neuroscience, 3, 24. https://doi.org/10.3389/neuro.09.024.2009

Pearson, J., Naselaris, T., Holmes, E. A., \& Kosslyn, S. M. (2015). Mental imagery: Functional mechanisms and clinical applications. Trends in Cognitive Sciences, 19(10), 590-602. https://doi.org/10.1016/j.tics.2015.08.003 
Rachman, S., \& de Silva, P. (1978). Abnormal and normal obsessions. Behaviour Research and Therapy, 16(4), 233-248. https://doi.org/10.1016/0005-7967(78)90022-0

Rassin, E. (2005). Thought Suppression. https://doi.org/10.1016/B978-0-08-044714-8.X5028-4

Salkovskis, P. M., \& Reynolds, M. (1994). Thought suppression and smoking cessation. Behaviour Research and Therapy, 32(2), 193-201. https://doi.org/10.1016/0005-7967(94)90112-0

Teasdale, J. D., Dritschel, B. H., Taylor, M. J., Proctor, L., Lloyd, C. A., Nimmo-Smith, I., \& Baddeley, A. D. (1995). Stimulus-independent thought depends on central executive resources. Memory \& Cognition, 23(5), 551-559. https://doi.org/10.3758/BF03197257

Teasdale, J. D., Proctor, L., Lloyd, C. A., \& Baddeley, A. D. (1993). Working memory and stimulusindependent thought: Effects of memory load and presentation rate. European Journal of Cognitive Psychology, 5(4), 417-433. https://doi.org/10.1080/09541449308520128

van Den Hout, M., Muris, P., Salemink, E., \& Kindt, M. (2001). Autobiographical memories become less vivid and emotional after eye movements. British Journal of Clinical Psychology, 40(2), 121-130. https://doi.org/10.1348/014466501163571

Versland, A., \& Rosenberg, H. (2007). Effect of brief imagery interventions on craving in college student smokers. Addiction Research and Theory, 15(2), 177-187.

https://doi.org/10.1080/16066350701200582

Vrana, S. R., Cuthbert, B. N., \& Lang, P. J. (1986). Fear imagery and text processing. Psychophysiology, 23(3), 247-253. https://doi.org/10.1111/j.1469-8986.1986.tb00626.x

Wang, D., Chatzisarantis, N. L. D., \& Hagger, M. S. (2017). Mechanisms underlying effective thought suppression using focused-distraction strategies: A self-determination theory approach. Psychology of Consciousness: Theory Research, and Practice, 4(4), 367-380. https://doi.org/10.1037/cns0000138

Wang, D., Chatzisarantis, N. L. D., \& Hagger, M. S. (2018). Why distractors with need-supportive content can mitigate ironic effects of thought suppression. Motivation and Emotion, 42(2), 214-224. https://doi.org/10.1007/s11031-017-9653-3

Wegner, D. M. (1994). Ironic processes of mental control. Psychological Review, 101(1), 34-52. https://doi.org/10.1037/0033-295x.101.1.34

Wegner, D. M., \& Erber, R. (1992). The hyperaccessibility of suppressed thoughts. Journal of Personality and Social Psychology, 63(6), 903-912. https://doi.org/10.1037/0022-3514.63.6.903

Wegner, D. M., Erber, R., \& Zanakos, S. (1993). Ironic processes in the mental control of mood and mood-related thought. Journal of Personality and Social Psychology, 65(6), 1093-1104. https://doi.org/10.1037/0022-3514.65.6.1093

Wegner, D. M., Schneider, D. J., Carter, S. R., \& White, T. L. (1987). Paradoxical effects of thought suppression. Journal of Personality and Social Psychology, 53(1), 5-13. https://doi.org/10.1037/0022-3514.53.1.5

Wenzlaff, R. M., \& Bates, D. E. (2000). The relative efficacy of concentration and suppression strategies of mental control. Personality and Social Psychology Bulletin, 26(10), 1200-1212. https://doi.org/10.1177/0146167200262003 
Wenzlaff, R. M., \& Wegner, D. M. (2000). Thought suppression. Annual Review of Psychology, 51(1), 59-91. https://doi.org/10.1146/annurev.psych.51.1.59

Whitaker, K. L., Watson, M., \& Brewin, C. R. (2009). Intrusive cognitions and their appraisal in anxious cancer patients. Psycho-Oncology, 18(11), 1147-1155.

https://doi.org/10.1002/pon.1512

The editorial and publishing process of this publication has been financed by the research grant "What is Thinking with Images?", SONATA 10, granted by the National Science Centre, Poland, on the basis of the decision No. 2015/19/D/HS1/02426. 